\title{
Research of Side Crash Sled Test based on Recursive Least Square Method
}

\author{
Dong Liping \\ School of Automotive Studies \\ Tongji University \\ Shanghai, China \\ donglipingshamo@163.com \\ Zhu Xichan \\ School of Automotive Studies \\ Tongji University \\ Shanghai, China \\ xczhu@163.com
}

\author{
Ma Zhixiong \\ School of Automotive Studies \\ Tongji University \\ Shanghai, China \\ mzx1978@163.com \\ Cao Yixing \\ School of Automotive Studies \\ Tongji University \\ Shanghai, China \\ caoyixing1989@163.comm
}

\begin{abstract}
In the vehicle side impact test, the dummy injuries were caused by the door intrusion. But because of the complexity of the door structure and the connection relation between the door and the vehicle body, the acceleration of the different position of the door was greatly diverse in the side impact. In order to make the velocity of different part of vehicle door in side crash sled test correlate to their velocity during vehicle crash test, the side crash sled test was equipped with different stiffness and damping. So, a lumped-mass model of side vehicle impact was built According to the regulation of occupant protection of vehicle side impact (GB20071-2006). And the spring stiffness and damping of different parts of vehicle door corresponding to the dummy abdomen and pelvis was estimated by the recursive least square method based on test data. Through verification the simulation result got a better accordance with experimental data.
\end{abstract}

Keywords- side crash; sled test; door intrusion velocity; lumped-mass model; recursive least square method (key words)

\section{INTRODUCTION}

In the vehicle side impact test, the dummy injuries were caused by the door intrusion. The intrusion velocity and timing at the position of the door corresponding to dummy chest, abdomen and pelvis directly affected the dummy injuries. Because of the complexity of the door structure and the connection relation between the door and the vehicle body, the acceleration of the different position of the door was greatly diverse in the side impact. As shown in Fig .1, there were the intrusion velocity curves in the position of the door corresponding to dummy chest, abdomen and pelvis. Although these curves had similar tendency, their peak and peak-time were varied greatly.

In the side impact test, the main contact between the door and dummy was the door trim which had three aspects resulting in the dummy injury: shape, stiffness and movement. However, for the deformation of each door region in the side impact was very different, the intrusion velocity and intrusion displacement of the door trim differed greatly in every contact region. The existing side impact sled test device could only reproduce one point's movement waveform of the door, but the simulation of the single point's intrusion was a little difficult to emerge the intrusion process of the whole door.

Therefore, a lumped-mass model of side vehicle impact was built According to the regulation of occupant protection of vehicle side impact (GB20071-2006). And the spring stiffness and damping of different parts of vehicle door corresponding to the dummy abdomen and pelvis was estimated by the recursive least square method based on test data.

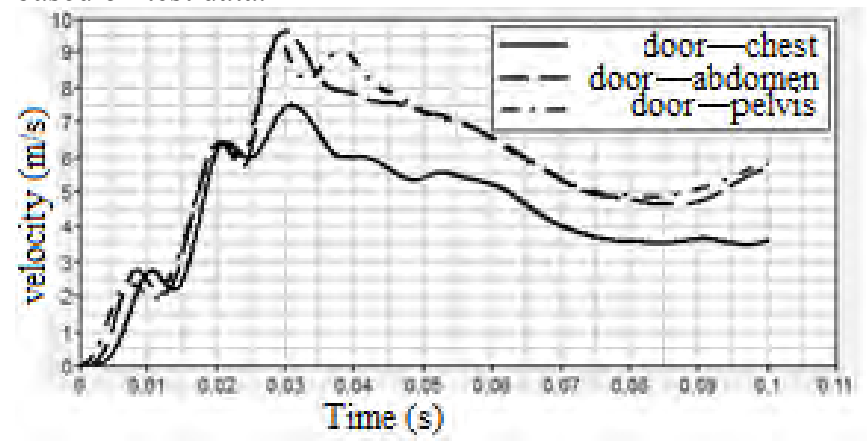

Figure 1. the intrusion velocity in the positions of the door corresponding to dummy chest, abdomen and pelvis

\section{ESTABLISHMENT OF THE SIDE IMPACT SIMULATION MODEL}

According to the regulation of occupant protection of vehicle side impact (GB20071-2006), the process of the entire side impact could be divided into two phases, namely: the first stage was that MDB (moving deformable barrier) came into contact with the door. The door panel was accelerated under the impact of MDB, while the outer door panel began to buckle from the extrusion of MDB. Then, the vehicle body was also pushed forward and the door system moved together with the vehicle body. Finally, 
the velocity of the whole test system (including the test vehicle, the dummy, MDB) was reduced to zero under the ground damping. Thus the vehicle side impact system was simplified as shown in Fig .2.

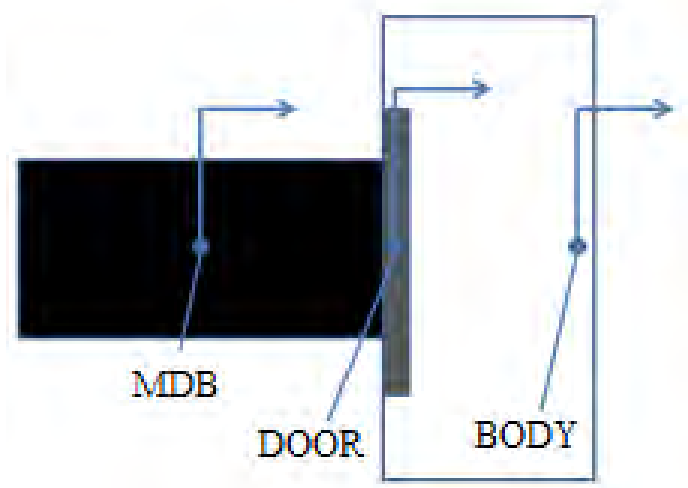

Figure 2. the diagrammatic sketch of the side impact

Through the above analysis, the side impact can be reconstructed by the lumped-mass model, such as shown in Fig .3. It consisted of three components in this model: $\mathrm{MDB}$, the door (including outer inner and inner door panel and the door trim) and the vehicle body. The connections among them were by the spring and the damp.

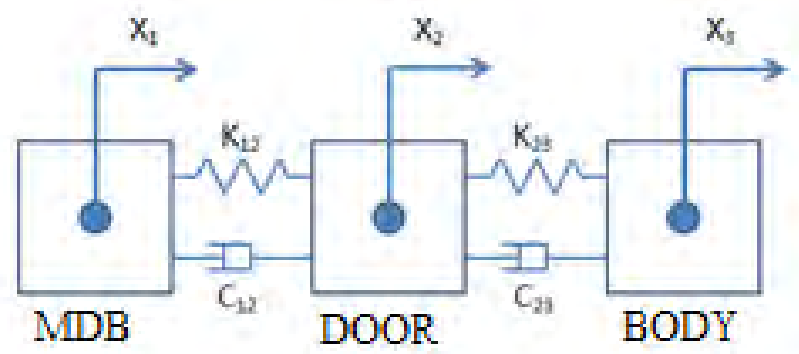

Figure 3. the lumped-mass model of side vehicle impact

In order to facilitate the following study, in the lumping-mass model some symbols were defined as follows: MDB's subscript was 1, door's was 2 and the body was 3 , and the mass of the three components was indexed with by $\mathrm{M}$, the stiffness of the spring was with $\mathrm{K}$ and the damping was $\mathrm{C}$. The stiffness of the spring between MDB and the door was $K_{12}$ and the damping was $C_{12}$; the stiffness of the spring between the door and the body was $K_{2 a}$ and the damping was $C_{2 \mathrm{a}}$.

So by the analysis on the force of MDB, door and body, the motion equation group can be expressed as follows:

$$
\left\{\begin{array}{c}
M_{1} \ddot{X}_{1}=-K_{12}\left(X_{1}-X_{2}\right)-C_{12}\left(X_{1}-\ddot{X}_{2}\right) \\
M_{2} \ddot{X}_{2}=K_{12}\left(X_{1}-X_{2}\right)-K_{2 a}\left(X_{2}-X_{a}\right)+C_{12}\left(X_{1}-X_{2}\right)-C_{2 a}\left(X_{2}-\ddot{X}_{a}\right) \\
M_{a} \ddot{X}_{a}=-K_{2 a}\left(X_{2}-X_{a}\right)+C_{2 a}\left(\dot{X}_{2}-\ddot{X}_{a}\right)
\end{array}\right.
$$

Among of them $\mathrm{X}$ donated the displacement of MDB, the door and the body, $X$ was their velocity and $\mathrm{x}$ was the acceleration.

The initial conditions were below:

$$
\left\{\begin{array}{c}
\dot{X}_{1}(0)=13.9 \\
X_{1}(0)=\ddot{X}_{1}(0)=0 \\
X_{2}(0)=\dot{X}_{2}(0)=\ddot{X}_{2}(0)=0 \\
X_{\pi}(0)=\ddot{X}_{\pi}(0)=\ddot{X}_{\pi}(0)=0
\end{array}\right.
$$

So, the equations could be resolved by using Duhamel's integral method if the spring stiffness and damping had already been known. However, for
$K_{12} 、 K_{2 a} 、 C_{12}, C_{2 a}$ they were greatly difficult to obtained from the side impact test. Hence, in this paper, a parameter estimation method was proposed based on the real impact test data. The system can be identified by means of parameter estimation in order to get the spring stiffness and damping in the test.

\section{PARAMETER ESTIMATION METHOD}

The equation in expression 1 can be converted in the form as follows

$M_{k} X_{k}=F_{x k}$ (the force related to the invasion displacement) $+F_{v k}$ (the force related to the invasion velocity) $k=1,2,3 \ldots$

Then it can define:

$$
\begin{aligned}
& F_{x k}=\sum_{i=1}^{n} K_{i} X_{i} \delta_{\mathrm{ki}} \\
& F_{v k}=\sum_{i=1}^{n} C_{i} X_{i} \delta_{\mathrm{ki}}
\end{aligned}
$$

And then the expression (3) could be changed as the equation (4).

$$
M_{k} X_{k}=\sum_{\substack{i=1 \\ k=1 \\ k=1}}^{n} K_{i} X_{i} \delta_{k i}+\sum_{i=1}^{n} C_{i} \hat{X}_{i} \delta_{k i}
$$

$\mathbb{X}_{k}$ was the acceleration of the mass lag numbered $k . X_{i}$ was the intrusion displacement of the spring numbered $i$ and $K_{\mathrm{i}}$ was the stiffness of the spring that can be taken as the coefficient of $X_{\mathrm{i}} \cdot X_{\mathrm{i}}$ was the intrusion velocity of the damping numbered $\mathrm{i}$.and $C_{\vec{i}}$ was the damping value that was the coefficient of $\dot{X}_{\mathrm{i}}$. $\delta_{\mathrm{ki}}$ was the related coefficient of the structure parameter and its values were set at $0 、 1 、-1$ according to the previous references.

The vehicle impact was a highly nonlinear process and the spring stiffness and damping were also the nonlinear change with the time during the impact. The causes may be divided into two aspects: the first was that both the relevant intrusion force involved with the intrusion displacement relative to the displacement and the relevant intrusion force involved with the intrusion velocity relative to the velocity were just as the nonlinear relationship. And the second was that the intrusion displacement and velocity in the time domain also constituted the nonlinear relationship. Thus, the coefficients $K_{\hat{\mathrm{i}}}$ and $C_{\tilde{i}}$ were also nonlinear in the time domain of the impact. However $K_{\hat{i}}$ and $C_{i}$ could be obtained by the online parameter estimation in the system identification.

\section{A. Recursive least square method}

In this paper, the unknown parameters $K_{12}, C_{12}, K_{2 a}$, and $C_{2 a}$ were estimated using the recursive least square method. The recursive least square method used less memory than the least square method and could realtimely identify the characteristics of dynamic system, so this recursive least square method was more suitable for this thesis's estimation model.

Because the system was a typical multi-input and multi-output system, the equation of the system must be discretized and an input and output difference model must be created.

$$
A(z) y(t)=B(z) u(t)+v(t)
$$

Where $y(t)=\left[y_{1}(t), y_{2}(t) w_{n}, y_{m}(t)\right]^{T} \in \mathbb{R}^{m}$ is the output variable matrix; 
$u(t)=\left[u_{1}(t), u_{2}(t), \ldots, u_{m}(t)\right]^{T} \in \mathbb{R}^{n}$ is the input variable matrix;

And $v(t)=\left[v_{1}(t), v_{2}(t), \cdots, v_{m}(t)\right]^{T} \in \mathbb{R}^{m}$ is the white noise vector;

$A(z)$ and $B(z)$ are the polynomial factor of the backward shift operator $z^{-1}$.

$$
A(z)=I+A_{1} z^{-1}+A_{2} z^{-2}+\cdots+A_{n_{a}} z^{-n_{n}} A_{i} \in \mathbb{R}^{m \times m}
$$$$
B(z)=B_{1} z^{-1}+B_{z^{-2}}+\cdots+B^{-n_{b}} z \in z^{m} \text { (8) }
$$

For the input and output difference model (5), if the expressions are defined as follows:

$$
\begin{aligned}
& \theta=\left[A_{1}, A_{2}, \cdots, A_{n_{a},} B_{1}, B_{2}, \cdots, B_{n_{b}}\right]^{T} \in \mathbb{R}^{m \times n}{ }_{a} n=m n_{a}+r n_{b}, \\
& \begin{aligned}
\varphi(t)= & {\left[-y^{T}(t-1),-y^{T}(t-2), \ldots{ }_{w}-y^{T}\left(t-n_{a}\right)_{s}\right.} \\
& \left.u^{T}(t-1), u^{T}(t-1), w_{v} u^{T}\left(t-n_{b}\right)\right]^{T} \in \mathbb{R}^{n}
\end{aligned}
\end{aligned}
$$

Then, the expression (7) can be transformed into the following model (11) :

$$
y(t)=\Phi(t) \theta+v(t)
$$

The model (11) is called the linear regression model of the multi-variable system.

Where $y(t)=\left[y_{1}(t), y_{2}(t), \cdots, y_{m}(t)\right]^{T} \in \mathbb{R}^{m}$ is the output variable matrix while $\Phi(t) \in \mathbb{R}^{m \times n}$ is called the regression information matrix including the input and output variables. And $\theta \in \mathbb{R}^{n}$ is the vector of the parameters needing to be identified while $v(t)=\left[v_{1}(t), v_{2}(t), \cdots, v_{m}(t)\right]^{T} \in \mathbb{R}^{m}$ is the white noise vector. The system can adopt a proper identification algorithm to reckon the unknown parameter vector $\theta$ by using the measured input and output data $(\{y(t), \Phi(t), t=1,2, \cdots\})$.

For the multi-variable regression identification model, the following criterion function can be defined and be minimized.

$$
j(\theta)=\Sigma_{j=1}^{t}\|y(j)-\Phi(j) \theta\|^{2}
$$

Because the system has kept $\mathrm{s}$ certain nonlinear, there will be some data saturation in the process of calculation using the least squares method. For the time-varying process, the data saturation will lead that the estimation value of the parameters cannot track the change of the time-varying parameters. In order to prevent the occurrence of data saturation, the forgetting factor $\mu$ $(0<\mu<1)$ is introduced and then the following index weighting matrix is constituted.

$$
\lambda=\operatorname{diag}\left[\mu^{N-1}{ }_{z} \cdots{ }^{2}, \mu^{N-1}, \mu, 1\right]
$$

This weighting scheme actually equaled focusing on the latest $1 /(1-\mu)$ observation data, so the former data before the estimated data has a little help to the parameter estimation. This can avoid the data saturation.

Thus, it can get the following recursive least square algorithm.

$$
\begin{gathered}
\tilde{\theta}(t)=\hat{\theta}(t-1)+K(t)[y(t)-\Phi(t) \hat{\theta}(t-1)] \\
K(t)=P(t-1) \Phi^{T}(t)\left[\mu I_{m}+\Phi(t) P(t-1) \Phi^{T}(t)\right]^{-1}
\end{gathered}
$$

$$
P(t)=\frac{1}{\mu}\left[I_{n}-K(t) \Phi(t)\right] P(t-1), P(0)=p_{0}
$$

Where $P(t) \in \mathbb{R}^{n \times n}$ is the covariance matrix and $K(t) \in \mathbb{R}^{\text {nxm }}$ is the gain matrix while $y(t)=\left[y_{1}(t), y_{2}(t), \cdots y_{m}(t)\right]^{T} \in \mathbb{R}^{m}$ is the output vector. $\Phi(t) \in \mathbb{R}^{m \times n}$ is the input and output regression matrix while $\hat{\theta}(t) \in \mathbb{R}^{n}$ is the being estimated parameter vector and $\mu$ is the forgetting factor.

\section{THE ANALYSIS ON THE CALCULATION RESULTS}

In this system, the mass parameters $M_{1 x} M_{2 x} M_{a}$ could be achieved by the measurement and the acceleration $\vec{X}_{1}, Z_{2}, Z_{a}$ could be obtained from the impact test. Taken the position of the door corresponding to the dummy chest for example, the accelerator $\vec{X}_{1}$ was fixed in the position of the MDB gravity and the accelerator $\vec{X}_{2}$ was set in the position of the door corresponding to the dummy chest and the accelerator $\vec{Z}_{a}$ was in the bottom of B pillar in the nonimpact side of the vehicle. Then the acceleration curves $\ddot{X}_{1}, \ddot{X}_{2}, \vec{X}_{a}$ were as shown in Fig .4 and from them it can obtain the relative intrusion displacement and velocity for both between MDB-door and door-body, as shown in Fig .5 and Fig .6. Then, the change of these unknown parameters in the test can be gotten by the recursive least square method.

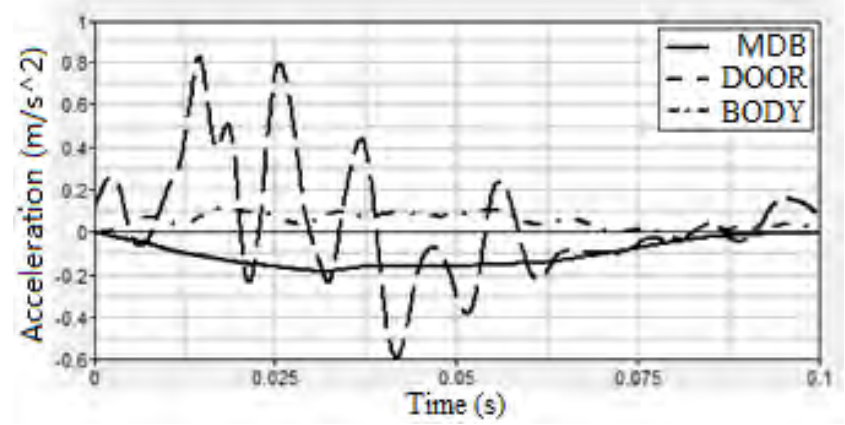

Figure 4. the acceleration curves of MDB, door and vehicle body

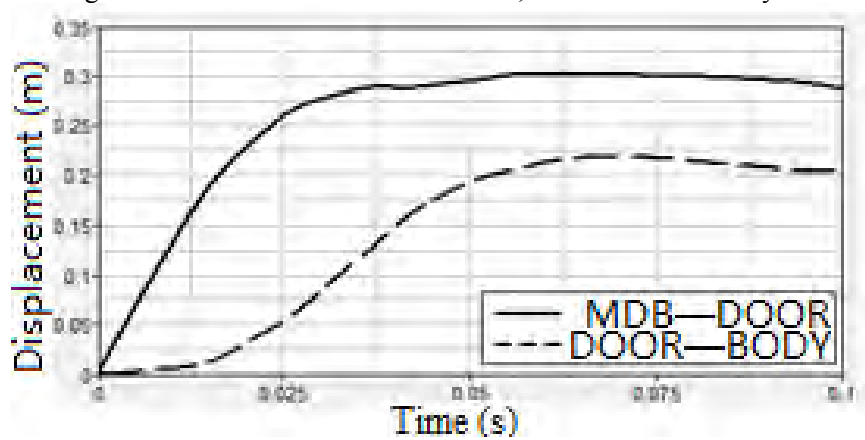

Figure 5 . the curve of the relative intrusion velocity

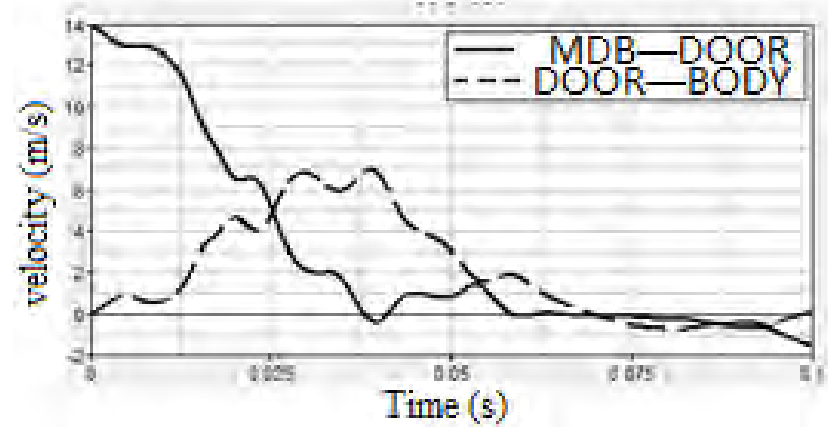

Figure 6. the curve of the relative intrusion displacement

Through the recursive least squares method with forgetting factor to estimate, the forgetting factor was taken as 0.98, and the initial value of the estimated parameters were such as the follows:

$$
\begin{gathered}
K_{12}(1)=K_{2 g}(1)=10^{6} \mathrm{~N} / \mathrm{m} \\
C_{12}(1)=C_{2 g}(1)=10^{2} \mathrm{~N} \cdot \mathrm{s} / \mathrm{m}
\end{gathered}
$$

Then, the results were as shown in Fig .7 to Fig .10. 


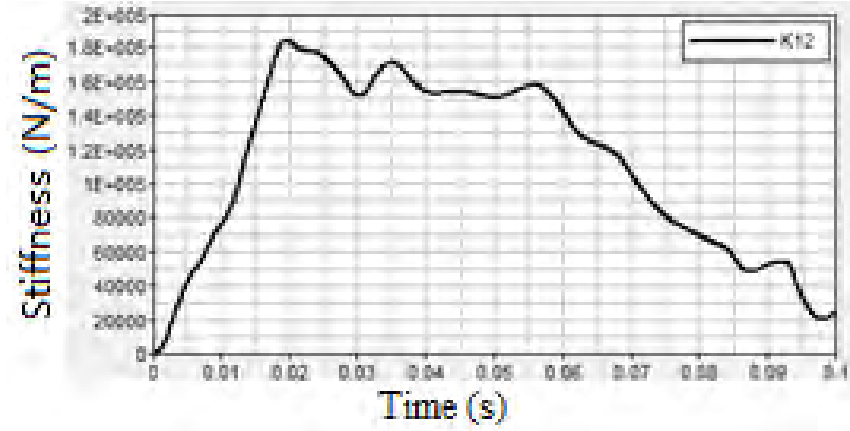

Figure 7. the curve of stiffness $K_{12}$ changing over time

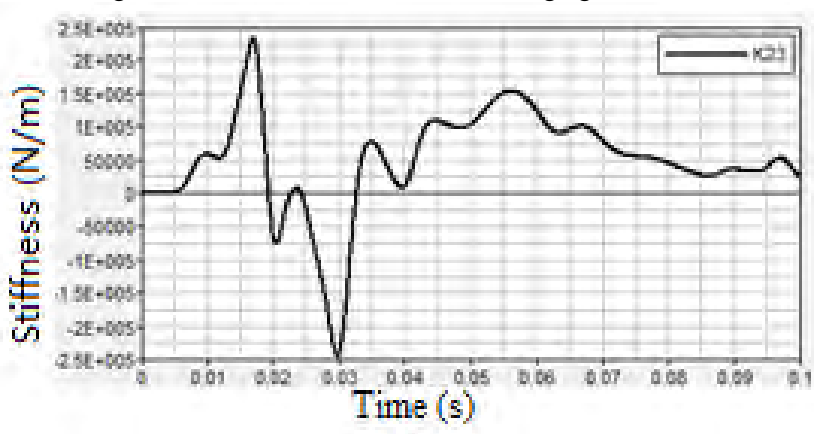

Figure 8. the curve of stiffness $K_{2 a}$ changing over time

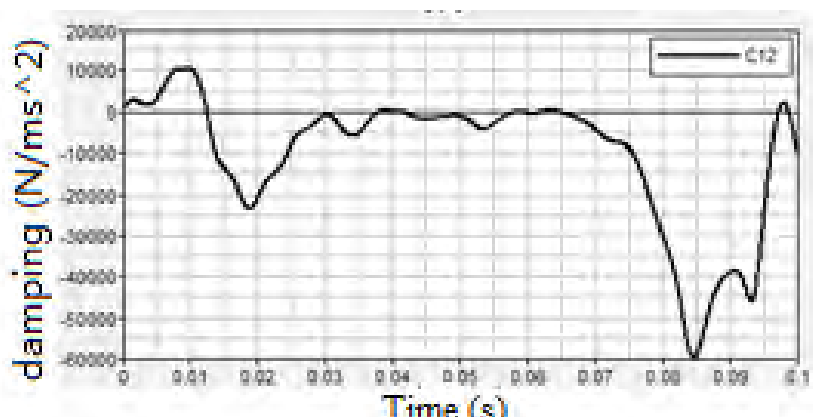

Figure 9. the curve of damping $C_{12}$ changing over time

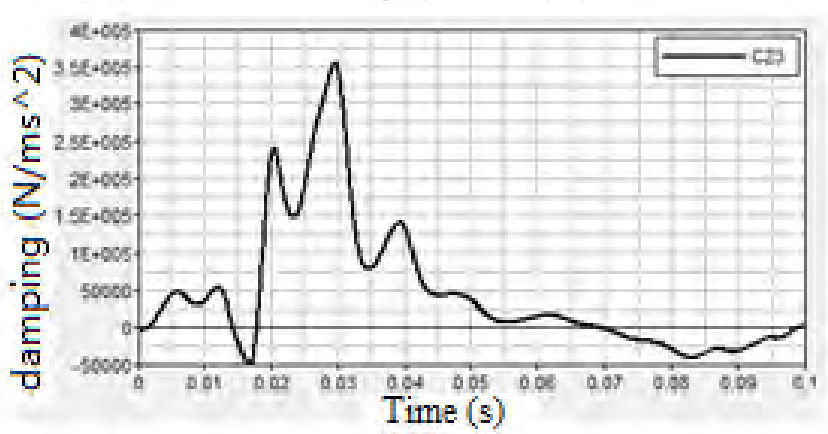

Figure 10. the curve of damping $C_{23}$ changing over time

Then taking the above results into the model (1) in chapter 2.1, and setting the timestep at $0.1 \mathrm{~ms}$, the total calculating time at $100 \mathrm{~ms}$ and the sampling points at 1000 , the simulation results were shown in Fig .11. From the comparison between the test result and simulation result in MDB door and body velocity, it can be seen that the simulation results were greatly consistent with the experimental results.

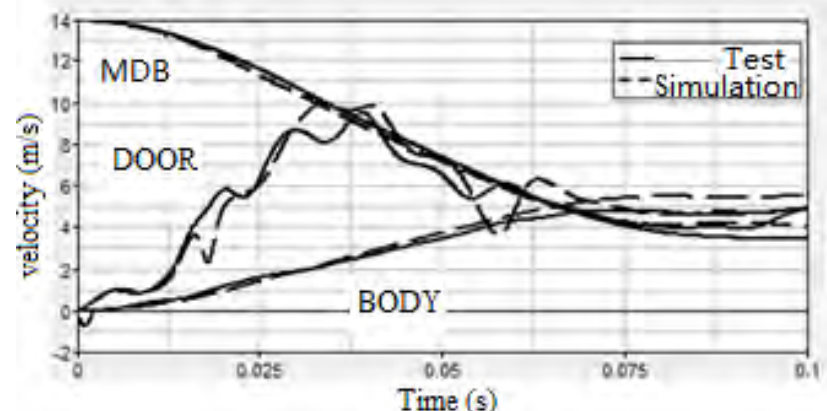

Figure 11. the comparison between test and simulation in velocity (doorchest)

It also can get the velocity in the positions of the door corresponding to the dummy abdomen and pelvis, as shown in Fig .12 and 13. It was proved that this method using the recursive least square method to get the stiffness and damping was accurate and effective.

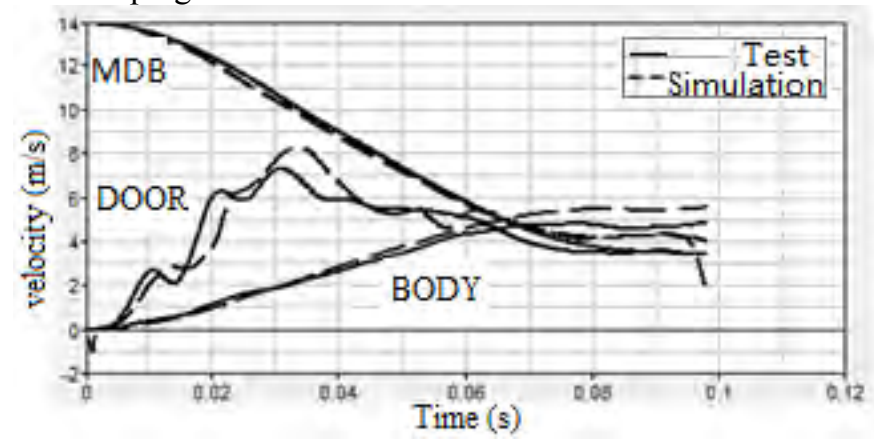

Figure 12. the comparison between test and simulation in velocity (door-

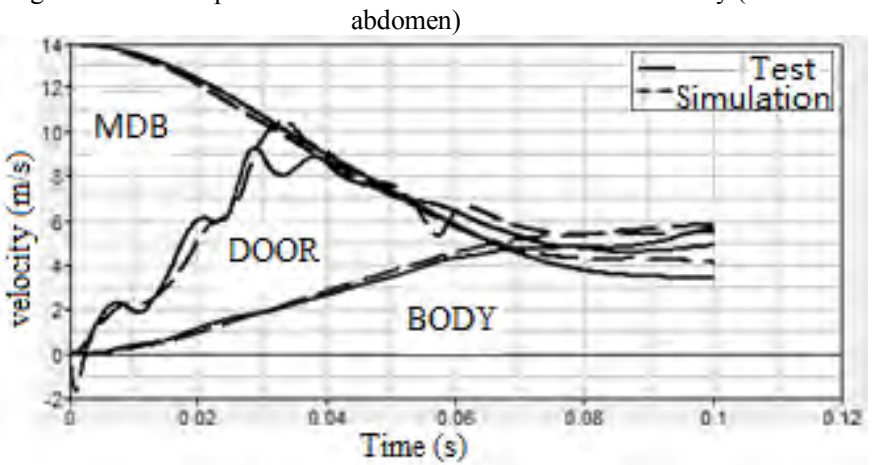

Figure 13. the comparison between test and simulation in velocity (doorpelvis)

\section{CONCLUSION}

The lumped-mass model was established based on the side impact of regulation GB20071-2006 in this paper. Based on the real test data, the unknown parameters (the stiffness and damping values changing over time) were estimated by using the least squares method with forgetting factors in the system. Finally, through the compare between the simulation result and the test data, this model was validated and got good consistency..

\section{REFERENCES}

[1] Zhang Jinhuan, Du Huiliang, and Ma Chunsheng, "Vehicle impact safety technologhy," Tsinghua University Press, 2010.

[2] Jayasuriya M M and Saha N K, "Local structural force evaluation of a vehicle," in International Journal of Crashworthiness, vol 10(5), 2005, pp. 451-461.

[3] U N Gandhi and S J HU, "Data-based approach in modeling," in Int. J. Impact Engng., vol. 16(1), 1995, pp. 95-118. 
[4] K. Aekbote, "A dynamic sled-to-sled test methodology for simulating dummy responses in side impact," in SAE, Paper No. 2007-01-0710, 2007.

[5] "Occupant protection of vehicle side impact," in China Regulation, NO. GB 2007-2006, 2006

[6] Paul Du Bois, and Clifford C. Chou, "Vehicle crashworthiness and occupant protection," American Iron and Steel Institute in press, 2004.
[7] Natthew Huang, "Vehicle crash mechanics," CRC Press,2002.

[8] Chou, C.C., Aekbote, K. and Le, J., "A review of side impact component test methodologies," in International Journal of Vehicle Safety, vol. 2, 2007, pp. 141-184.

[9] Claeson, N. and Ryrberg, S., "The door mounted inflatable curtain," in SAE, Paper No. 2006-01-1437, 2006.

[10] TRO, "MADYMO theory manual version 7.3," TNO Road Vehicle Research Institute, 2012. 